CLINICAL STUDY

\title{
Quality of life is reduced in patients with Klinefelter syndrome on androgen replacement therapy
}

\author{
Willem de Ronde, Anne de Haan and Madeleine L Drent \\ Department of Endocrinology, Vrije Universiteit Medical Center, 1081 HV Amsterdam, The Netherlands \\ (Correspondence should be addressed to W. de Ronde; Email: p.deronde@vumc.nl)
}

\begin{abstract}
Context: Klinefelter syndrome (KS) is a genetic disorder, characterized by an XXY karyotype, hypergonadotrophic hypogonadism and infertility, which affects $\sim 1$ in 700 men. KS has also been associated with neuropsychological alterations.

Objective: To investigate whether quality of life (QoL) is reduced in patients with $\mathrm{KS}$ on androgen treatment.

Design: Observational study.

Methods: Questionnaires (RAND-36) were sent to $46 \mathrm{KS}$ patients on androgen treatment who regularly visit the outpatient clinic of an academic medical centre. QoL scores were compared with a Dutch male reference group.

Results: Forty (87\%) questionnaires were returned which were suitable for evaluation. KS patients had significantly lower (worse) scores on eight of the nine domains of the RAND questionnaire compared with the male reference group. In KS patients, higher education was associated with significantly better QoL scores.

Conclusions: QoL is reduced in patients with KS on androgen treatment.
\end{abstract}

European Journal of Endocrinology $160465-468$

\section{Introduction}

In 1942, Harry Klinfelter et al. described a syndrome characterized by small testes, infertility and gynaecomastia later named Klinefelter syndrome (KS) (1). KS is a genetic disorder, characterized by a 47 , XXY karyotype, which affects $\sim 1$ in 700 men (2). Although the clinical presentation of $\mathrm{KS}$ is heterogeneous, the syndrome is characterized by testicular dysfunction resulting in androgen deficiency and infertility in practically all patients (3). KS has also been associated with neuropsychological alterations (4). Some of these disorders might be the result of late or insufficient androgen exposure during puberty. It has been our clinical experience that patients with KS frequently report psychological or physical discomfort that is not relieved by an adequate androgen treatment. Therefore, we wanted to investigate whether quality of life (QoL) is reduced in patients with KS on androgen treatment.

\section{Subjects and methods}

The department of endocrinology of the VU University Medical Center is a tertiary referral centre for andrological problems. We have sent questionnaires to all patients with a diagnosis of KS who are on androgen replacement therapy and regularly visit our outpatient clinic. This study was approved by the Medical Ethics Committee of the Amsterdam VU University Medical Center, and all volunteers gave written informed consent before receiving the questionnaire. Questionnaires included questions about age, educational level and duration of androgen therapy. Educational level was classified as lower (primary school and lower professional training), middle (secondary school or middle professional training) and higher (bachelor or master). We used the RAND-36 health survey to score QoL because it is a simple, reliable and reproducible test. The RAND-36 survey comprises nine subscales evaluating i) physical limitations in daily life, ii) social limitations resulting from health problems, iii) limitations with work or daily activities resulting from physical problems, iv) similar limitations resulting from emotional problems, v) mental health, vi) vitality, vii) pain, viii) general health perception and ix) health change. High scores indicate better QoL. The scores of the respondents were compared with published reference values from a Dutch male population-based sample $(5,6)$. This sample comprised 372 males, aged between 
18 and $89(44.1 \pm 17.5)$ years. Out of this sample, $42 \%$ received lower education, $40 \%$ middle education and $18 \%$ higher education.

\section{Statistical analysis}

All results are described as frequencies, means \pm s.D. and percentages. Differences between the KS group and the reference population were tested for significance using an unpaired Students $t$-test for normally distributed variables. We used multiple linear regression analysis to study the impact of education, age and duration of treatment on the scale scores and to study the relationship between education and age at which androgen treatment was started. We used a general linear model to investigate the impact of education class or type of androgen treatment on the combined scores using scale number as the independent variable (within subjects factor), scale score as the dependent variable and education or type of androgen treatment as between-subject factor. Associations between type of androgen treatment and education were tested for significance using a Kruskall-Wallis test.

\section{Results}

From the 46 distributed questionnaires, 42 (91\%) were returned out of which $40(87 \%)$ were appropriate for evaluation. The characteristics of the respondents are presented in Table 1. Mean age and educational level were not significantly different between the KS group and the male reference group.

In Table 2, the RAND-36 scores are presented for the KS patients and a male Dutch reference group. The KS patients had significantly lower (worse) scores on eight out of nine subscales compared with the general population. In a multiple regression model, educational level in KS patients was significantly and positively associated with RAND scores for the scales vitality $(P=0.008)$ and general health $(P=0.009)$. Age and

Table 1 Baseline characteristics of respondents and a male reference sample.

\begin{tabular}{lll}
\hline$n$ & 40 & 372 \\
Age (years) & $46 \pm 11.8$ & $44 \pm 17.5$ \\
Maximal education (\%) & & \\
$\quad$ Lower education & 43 & 42 \\
Middle education & 35 & 40 \\
Higher education & 23 & 18 \\
Current therapy & & \\
Injections (\%) & 23 & \\
Mean dose (mg/week (range)) & $73(17-125)$ & \\
Oral (\%) & 8 & \\
Mean dose (mg/day (range)) & $240(160-320)$ & \\
Gel (\%) & 70 & - \\
$\quad$ Mean dose (mg/day (range)) & $55(25-100)$ & \\
Duration of treatment (years) & 12.6 & \\
\hline
\end{tabular}

Table 2 RAND scores in patients with Klinefelter syndrome (mean \pm S.D.) and a male reference group.

\begin{tabular}{lccl}
\hline RAND subscale & KS & Male reference & $\boldsymbol{P}$ value \\
\hline Physical functioning & $74 \pm 26$ & $85 \pm 22$ & $<0.05$ \\
Social functioning & $59 \pm 30$ & $88 \pm 20$ & $<0.001$ \\
Role physical & $56 \pm 42$ & $82 \pm 34$ & $<0.001$ \\
Role emotional & $65 \pm 44$ & $87 \pm 29$ & $<0.01$ \\
Mental health & $66 \pm 19$ & $79 \pm 17$ & $<0.001$ \\
Vitality & $51 \pm 25$ & $70 \pm 21$ & $<0.001$ \\
Pain & $63 \pm 35$ & $83 \pm 24$ & $<0.01$ \\
General health & $56 \pm 29$ & $71 \pm 23$ & $<0.01$ \\
Health change & $58 \pm 30$ & $53 \pm 18$ & NS \\
\hline
\end{tabular}

duration of treatment were not significantly associated with the scores on any scale. In Fig. $1 \mathrm{a}$ and $\mathrm{b}$, the mean RAND scores are summarized and grouped according to education level and type of androgen treatment. Subjects who received higher education have consistently and significantly higher mean scores compared with subjects who have lower or middle education $(P=0.03$ with lower and middle groups combined versus higher education). The mean scores for the users of testosterone gel are significantly lower compared with those of oral or injectable testosterone users $(P=0.03)$. Education was not significantly associated with the type of androgen treatment or age at the start of androgen treatment.

\section{Discussion}

In this study, we compared the QoL in KS patients with a reference database of the general population. In the outpatient clinic, we are frequently confronted with KS patients with physical, rather non-specific symptoms. Regularly these symptoms are attributed by the patient to the use of androgen replacement and it is believed that changing the dose or type of androgen replacement might relieve these physical problems. In our study, KS patients showed significantly lower scores on physical, social and psychological scales. KS is characterized by infertility and hypogonadism. Hypogonadism may be associated with physical discomfort but it is believed that most of these complaints will disappear after a period of appropriate androgen replacement. The KS patients in our study had androgen replacement for a mean period of 12.6 years and it can be assumed that most physical consequences of hypogonadism are largely corrected after this period. Irrespective of the androgen replacement, KS has been associated with a higher prevalence of certain types of disease in large case-control studies $(7,8)$. However, the prevalence of these diagnoses was low and cannot explain the overall lower QoL in our subjects. It can be assumed that the physical discomfort as reported by our subjects largely reflects a generally lower psychological and social sense 

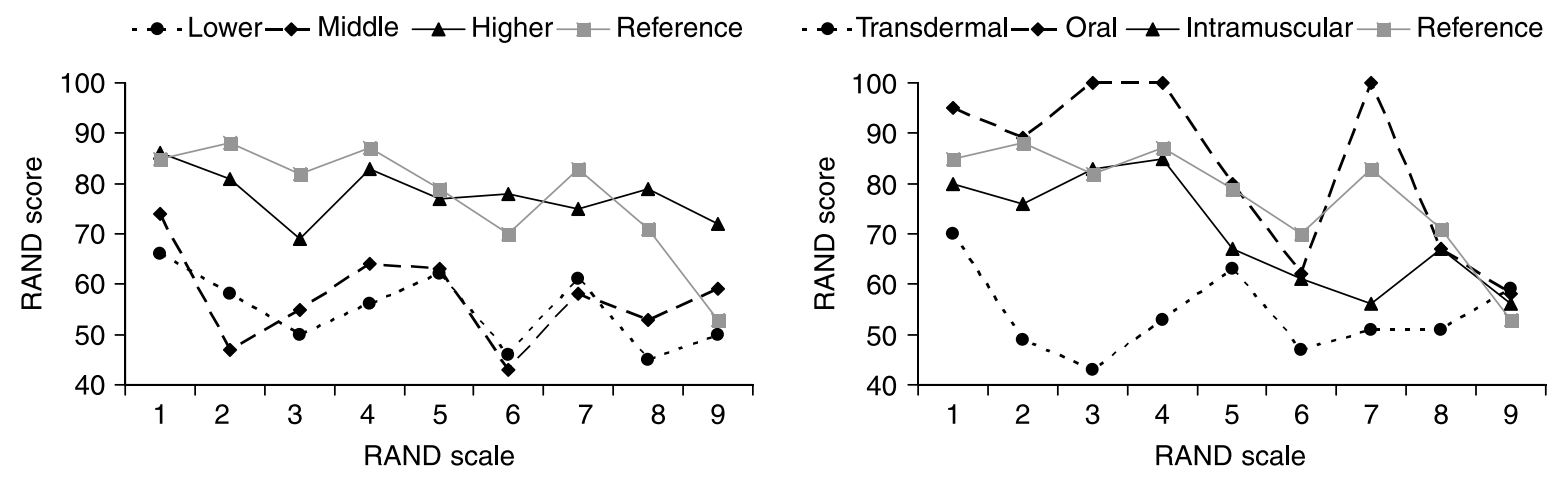

Figure 1 ( $a$ and $b)$ Mean QoL scores in KS patients stratified by educational level (left) or type of androgen treatment (right).

of well-being as is shown in our study. Indeed, it has been shown in previous studies that KS is associated with more difficulties in identifying and verbalizing emotions (9). Moreover, patients with KS appeared to be more easily emotionally aroused (10). The same investigators reported a higher prevalence of psychiatric disorders in KS patients compared with the general population (11).

The reference population in our study is a populationbased sample from Dutch males of which the results were previously published (5). Unfortunately, no education stratified results of this sample were published. However, mean age and educational level were not significantly different for the studied KS patients and the reference group. KS patients with higher education reported significantly higher QoL scores compared with those with middle or lower education. Also in the general population, educational level is positively associated with RAND scores on the scales of physical functioning and general health (6). In KS patients with a bachelor or master degree, QoL scores were similar to those of the reference population; however, the reference population had a much lower mean level of education. Perhaps higher educated KS patients are better equipped to deal with the sequelae of KS. Alternatively, higher education may be a result of a less severe phenotype. A less severe phenotype may be associated with a later age at which the androgen treatment is started; however, in our study, educational level and age to start the androgen treatment were not significantly associated.

Surprisingly, the users of testosterone gel reported significantly lower QoL scores compared with oral or injectable testosterone users. Only 3 out of 40 patients used oral testosterone-undecanoate and 9 out of 40 used i.m. injections. Since KS patients in this study were not randomized to the type of androgen treatment, it is impossible to draw conclusions regarding a possible causal relationship between the use of testosterone gel and lower QoL. Testosterone gel has been proven to be a safe and effective mode of testosterone treatment, providing relatively stable and physiological testosterone levels in the majority of users $(12,13)$. The pharmacokinetic profile is more physiological compared with oral testosterone-undecanoate (14) or i.m. injections $(15,16)$. Because of the better profile, it may be speculated that some patients switched to testosterone gel when it became available in the Netherlands (2003) in an attempt to relieve psychological or physical complaints that remained or were attributed to the use of oral or i.m. testosterone.

Our study has several limitations. Although we assume that androgen treatment has a positive effect on QoL in KS patients with low plasma testosterone levels, we were unable to quantify this postulated effect due to the fact that we did not include a non-androgentreated group of KS patients in our study. All subjects in the study were under active surveillance at our outpatient clinic and received optimal care. However, the adequacy of androgen treatment during the study was not confirmed by biochemical measures such as plasma testosterone levels. The number of studied KS patients was relatively small and the patients were recruited from a tertiary referral centre that is likely to attract more problematic patients. Therefore, the results of this study need to be confirmed in a larger, more heterogeneous KS population to increase external validity. Additional research is needed to address the causes of the lower QoL in these patients in order to optimize the care for KS patients.

In conclusion, our results show that patients with KS on average have a lower QoL. They report more physical, emotional and social problems compared with the general population. Our results do not support the assumption that these problems may resolve after the start of androgen treatment. Possibly, due to their problematic verbalization of emotions, KS patients are more likely to express their lower sense of well-being as a physical problem.

\section{Declaration of interest}

The authors declare that there is no conflict of interest that could be perceived as prejudicing the impartiality of the research reported. 


\section{Funding}

This research did not receive any specific grant from any funding agency in the public, commercial or not-for-profit sector.

\section{References}

1 Klinefelter HF. Klinefelter's syndrome: historical background and development. Southern Medical Journal 198679 1089-1093.

2 Bojesen A, Juul S \& Gravholt CH. Prenatal and postnatal prevalence of Klinefelter syndrome: a national registry study. Journal of Clinical Endocrinology and Metabolism 200388 622-626.

3 Lanfranco F, Kamischke A, Zitzmann M \& Nieschlag E. Klinefelter's syndrome. Lancet $2004 \mathbf{3 6 4} 273-283$.

4 Boone KB, Swerdloff RS, Miller BL, Geschwind DH, Razani J, Lee A, Gonzalo IG, Haddal A, Rankin K, Lu P \& Paul L. Neuropsychological profiles of adults with Klinefelter syndrome. Journal of the International Neuropsychological Society 2001 7 446-456.

5 VanderZee KI, Sanderman R \& Heyink J. A comparison of two multidimensional measures of health status: the Nottingham Health Profile and the RAND 36-Item Health Survey 1.0. Quality of Life Research 19965 165-174.

6 VanderZee KI, Sanderman R, Heyink JW \& de HH. Psychometric qualities of the RAND 36-Item Health Survey 1.0: a multidimensional measure of general health status. International Journal of Behavioral Medicine 19963 104-122.

7 Bojesen A, Juul S, Birkebaek N \& Gravholt CH. Increased mortality in Klinefelter syndrome. Journal of Clinical Endocrinology and Metabolism 200489 3830-3834.

8 Swerdlow AJ, Higgins CD, Schoemaker MJ, Wright AF \& Jacobs PA. Mortality in patients with Klinefelter syndrome in Britain: a cohort study. Journal of Clinical Endocrinology and Metabolism 200590 6516-6522.
9 van Rijn S, Aleman A, Swaab H, Krijn T, Vingerhoets G \& Kahn R. What it is said versus how it is said: comprehension of affective prosody in men with Klinefelter $(47, \mathrm{XXY})$ syndrome. Journal of the International Neuropsychological Society 200713 1065-1070.

10 van Rijn S, Swaab H, Aleman A \& Kahn RS. X Chromosomal effects on social cognitive processing and emotion regulation: a study with Klinefelter men (47,XXY). Schizophrenia Research 200684 194-203.

11 van Rijn S, Aleman A. Swaab H \& Kahn R. Klinefelter's syndrome (karyotype 47,XXY) and schizophrenia-spectrum pathology. British Journal of Psychiatry $2006 \mathbf{1 8 9}$ 459-460.

12 Swerdloff RS, Wang C, Cunningham G, Dobs A, Iranmanesh A, Matsumoto AM, Snyder PJ. Weber T, Longstreth J \& Berman N. Long-term pharmacokinetics of transdermal testosterone gel in hypogonadal men. Journal of Clinical Endocrinology and Metabolism $2000854500-4510$

13 Wang C, Cunningham G, Dobs A, Iranmanesh A, Matsumoto AM, Snyder PJ, Weber T, Berman N, Hull L \& Swerdloff RS. Long-term testosterone gel (AndroGel) treatment maintains beneficial effects on sexual function and mood, lean and fat mass, and bone minera density in hypogonadal men. Journal of Clinical Endocrinology and Metabolism 200489 2085-2098.

14 Bagchus WM, Hust R, Maris F, Schnabel PG \& Houwing NS. Important effect of food on the bioavailability of oral testosterone undecanoate. Pharmacotherapy 200323 319-325.

15 Conway AJ, Boylan LM, Howe C, Ross G \& Handelsman DJ. Randomized clinical trial of testosterone replacement therapy in hypogonadal men. International Journal of Andrology 198811 247-264.

16 Cantrill JA, Dewis P, Large DM, Newman M \& Anderson DC. Which testosterone replacement therapy? Clinical Endocrinology 198421 97-107.

Received 16 December 2008

Accepted 3 January 2009 